Monatsschrift f. Geburtshülfe u. Gynäkologie 1926;75:I-II

\title{
Contents, Vol. 75, No. 1-2, 1926
}

\section{Inhaltsverzeichnis}

Seite

Zum Geleit 1

Wíssenschaftlîche Arbeîten und Veröffentlíchiingen. Von Paul

Straßmann 2

Mißlungene geburtshilflîche Eingriîfe. Von Geh.-Rat Prof. Dr.

\section{Stoeckel-Beriin 7}

Sterilität und Konstïtution. Von Prof. Dr. A. Mayer-Tübingen . . 21 Die Gelenkerkranku $\pi$ gen

während der Klimax. Von Geh.-Rat Prof.

W. $\mathrm{j}^{7} / 8 \mathrm{~s} \cdot$ Berlin . $\quad 26$

Über familiäres Auftrete $\pi$ von angeborenen Mißbildungen. Von

Geh.-Rat Prof. Dr. Otto Hildebrand-Beńin 32

Metrorrhagîen aus Thrombopenie und îhre Behandlung. Von Geh.-

Rat Prof. Dr. Georg Klemperer-Bt门́m

35

Zur Beurteilung der Keimdrüsenpräparate. Von Geh.-Rat Prof. Dr.

C. Posner-Berlin 42

Der gegenwärtíge Stand der Lungenprobe. Von Geh.-Rat Prof. Dr.

F. Straßmann-Berl'm 47

Pertubation und Salpingostomîe zur Beseitigung der Sterilität. Von

Dr. H. Fuchs-Oanzig 56

Zur Frage des Hallux valgus. Von Prof. Dr. Richard Mühsam-Beńin 65

Die Operation des Prolapses und der Retroflexio uteri fixata. Von

Dr. Gotthard Schubert-Beuiben

Die Bedeutung der Zystoradiographie îür die Diagnostik der Harn-

röhren- und Blasenfisteln. Von San.-Rat Dr. Arthur Lewin-

Berlin 76

Brauchbare und unbrauchbare Abtreibungsmittel. Von Med.-Rat Dr.

Georg Straßmann-Bresia,u 79

Primäres Ureterkarzinom. Von Dr. Hans L. Posner-jüterbog . . 86

Zur Frage über die Nierenausschaltung mittels Ureterknotung nach

Ureterresektion. Von Prof. Dr./. Braude-1·Aoskau .... 90

Experimentelle Untersuchungen zur Frage über die Ureterknotung.

Von Dr. E. M. Schwarsmann-Moskm

103

Pyelitis gravidarum. Von Dr. Paul Meyer-BtxWn 109

Eine angeborene kystomartige Bildung des rechten Ovarium. Von

Dr. Hans-Otto Neumann-Marburg 123 Arbeiten aus der Straßmannschen KПniki seite

Über den Begriff des Uterus pseudounicornis. Von Dr. Martin

Jung-Y!tïWii

131

Die Naegele-1\& $\Lambda$ g $\varnothing$ am Steiß. Von Dr. G. E. $\varnothing_{i}^{3 / 8^{1} / 8-B e r l i n} \ldots 143$ 
Uber einen Fall von kombinierter Hemmungsmißbildung. Von

Dr. Albert Sf $\varnothing^{7} / 8 /$-Berlin

150

Uber Ròntgenbestrahlung bei Myomen. Von Dr. Gerhard Zimmer-

Berlin

157

Die Lumbalanästhesie bei Sectio caesarea. Von Dr. Ernst Fried-

mann-BerYm 163

Die Ursache der Arkadie. Von Or.Erwin Straßmann-BzrYm . .172

Schlußwort. Von Geh.-Rat Prof. Dr. A. Martin

187

Die gynäkologische Strahlentherapie im Jahre 1925. Sammelbericht

von Dr. C. H. Engelbrecht-EvL·ngeh ...

189

Breslauer Gynäkologische Gesellschaft. Sitzung vom Id.Juni 1926209

Buchanzeigen

227

Literatur-Verzeichnís 